\title{
Integrating Electronics and Microfluidics on Paper
}

\section{Citation}

Hamedi, Mahiar M., Alar Ainla, Firat Güder, Dionysios C. Christodouleas, M. Teresa FernándezAbedul, and George M. Whitesides. 2016. Integrating Electronics and Microfluidics on Paper. Advanced Materials 28, no. 25: 5054-5063. Portico. doi:10.1002/adma.201505823.

\section{Published Version}

10.1002/adma.201505823

\section{Permanent link}

http://nrs.harvard.edu/urn-3:HUL.InstRepos:29953305

\section{Terms of Use}

This article was downloaded from Harvard University's DASH repository, and is made available under the terms and conditions applicable to Open Access Policy Articles, as set forth at http:// nrs.harvard.edu/urn-3:HUL.InstRepos:dash.current.terms-of-use\#OAP

\section{Share Your Story}

The Harvard community has made this article openly available.

Please share how this access benefits you. Submit a story.

Accessibility 
DOI: $10.1002 /(($ please add manuscript number $))$

Article type: Communication

\section{Integrating Electronics and Microfluidics on Paper}

Mahiar M. Hamedi, Alar Ainla, Firat Güder, Dionysios C. Christodouleas, M. Teresa Fernández-Abedul, George M. Whitesides ${ }^{*}$

Dr. M. M. Hamedi, Dr.A. Ainla, Dr. F. Guder, Dr. D. C. Christodouleas, Prof. G. M. Whitesides

Department of Chemistry and Chemical Biology, Harvard University, Cambridge MA, USA

Prof. T. Fernández-Abedul

Departamento de Química Física y Analítica, Universidad de Oviedo, Julián Clavería 8, 33006 Oviedo, Asturias, Spain

Prof. G. M. Whitesides

Kavli Institute for Bionano Science and Technology, Harvard University, Cambridge, MA 02138, USA

Wyss Institute for Biologically Inspired Engineering, Harvard University, Cambridge, MA, USA

(*) Author to whom correspondence should be addressed:

gwhitesides@gmwgroup.harvard.edu 
Integrated systems incorporating fluidic and electronic components have the potential to generate new functions and devices, especially in micro total analysis systems ( $\mu \mathrm{TAS})$. Today, the majority of $\mu \mathrm{TAS}$ are constructed by stacking electronic structures, (which are formed on flat substrates) and microfluidic structures (usually made in glass, or organic polymers). ${ }^{[1]}$ These stacked systems have the disadvantage that they require multiple steps of fabrication, assembly, mounting, and connection. ${ }^{[2]}$ Integration of $\mu \mathrm{TAS}$ in paper has the potential to minimize the design complexity of systems, and to enable scalable manufacturing via printing. The integration of printed electronic and microfluidics on paper is, however, in its early stage of development. ${ }^{[3]}$ The first electroanalytical paper devices, combined paper microfluidics with electrochemistry using screen-printed electrodes. ${ }^{[4]}$ Since then, a wide range of different electrochemical paper devices have been reported by us and others. ${ }^{[5-13]}$

In one widely used approach to paper microfluidics hydrophobic barriers are patterned into paper ${ }^{[14],[15]}$ - usually using wax printing - to direct the flow of fluids by capillary wicking. ${ }^{[16]}$ In paper-based electronics, ${ }^{[17]}$ thin films of electronic materials are usually patterned by direct printing. ${ }^{[17-20]}$ Paper microfluidics and printed electronics have historically developed separately, and the techniques developed for their fabrication, are usually incompatible. Several factors contribute to this incompatibility, including different objectives and methods of fabrication: paper microfluidics transports water, while printed electronics transports electrons. In printed electronics, functional structures (e.g. conductors) are printed on the surface of the paper, while in paper microfluidics, structures are created in the interior of the paper substrate (e.g. as hydrophobic barriers, or regions to contain biomolecules or reagents). This incompatibility has made full integration of microfluidic and electronic systems in paper impossible. More specifically, the current paper-based 
microfluidic electrochemical systems, have five major limitations: i) Complex Interconnects. Surface-printed conductors cannot be connected between different layers of paper without cutting via holes and registering the patterns on different layers. ii) Reduced Fluid Flow. Conventionally printed carbon and silver electrodes are hydrophobic. The hydrophobicity can slow, or stop, the flow of aqueous liquids that pass through or adjacent to the ink. iii) Limited Surface Area. Carbon and silver inks (which are viscous) do not penetrate inside the paper substrate, and thus do not exploit the interior surface area of paper. iv) Poor Contact Adhesion and Stability. Carbon inks, when printed on top, do not adhere well to bare paper, and smaller structures can crack or tear during handling, folding or swelling of the paper. v) Blocking of the Pores in the Paper. Aqueous solutions cannot pass through electrodes printed on top of paper. Components that are simultaneously electrical and fluidic, therefore, cannot be fabricated. This limits the design of electrochemical devices; for example, designs that require several electrode layers stacked on top, cannot easily be made.

This paper demonstrates a technique that overcomes all five of these limitations by co-fabricating electronic and microfluidic structures on paper via printing. In more detail: the procedure for co-fabrication starts with a channel formed by printing of wax, and melting it into the paper. ${ }^{[16]}$ The channels can define either fluidic or electronic structures (Figure 1B). The extension of this technique to make electrically conducting wires relies on water-dispersed electrically conducting inks, since these inks can wick inside paper microfluidic channels. We used the waterdispersed conducting polymer mixture poly(3,4-ethylenedioxythiophene)polystyrene sulfonate (PEDOT), and water-dispersed multiwall carbon nanotubes (MWNTs) as inks. The inks were added to the wax-defined channels that had been designed to 
become electrically conducting wires, and spread by wicking to cover the entire hydrophilic area of the paper. On drying, the inks coated the cellulose fibers inside the paper and formed electrically conducting wires. Remarkably, the ink covered with fibers without blocking the pores of paper, and left the channels hydrophilic. We used PEDOT because it has high electrical conductivity $(>1000 \mathrm{~S} / \mathrm{cm}),{ }^{[21]}$ and is stable in water and air. ${ }^{[22]}$ MWNTs were used because the electronic and electrochemical behavior of MWNTs resemble that of the graphite flakes ${ }^{[23]}$ that constitute the commonly used carbon inks. MWNTs, however, have a high aspect ratio (>1000), and can form strong films that can be bent without cracking; They also form electrically conducting percolation networks at lower concentrations than carbonblack nanoparticles. We used carboxymethylcellulose to disperse the MWNTs in water for making the inks. ${ }^{[24]}$ Figure $1 \mathrm{C}$ shows a photograph in which the two different conducting inks, PEDOT (blue) and MWNT (black) have been patterned in different parts of the paper microfluidic structure. Both of these conductors penetrate the full thickness of the paper (Figure 1C). It is, therefore, straightforward to connect points on opposite sides of the paper, and complex 3D circuits can be fabricated by stacking several sheets of paper. More importantly, the combination of open pores, and hydrophilic surfaces, implies that aqueous solutions can wick through the electrical conductors. These structures then form both electronic (defined by the conducting paths) and fluidic conductors (defined by the wax barriers). For this reason we call them "electrofluidic" structures or channels. In most electrochemical device designs, it is also necessary to have "conventional" water-impermeable conductors to form connection points to instruments. We fabricated waterimpermeable conductors simply by using a second step of wax printing. In the regions where the second wax layer overlapped with the electrofluidic patterns (Figure 1A, 
1B), the wax melted and formed a hydrophobic layer around the conducting films deposited on the cellulose fibers. After this process, the channels would still conduct electricity, but were not wetted by water, and would not sustain flow of aqueous solutions driven by capillarity.

In summary, the method we describe allows co-fabrication of four different components using printing (Figure 1C): i) fluidic and electrical insulators comprising paper coated only with hydrophobic wax; ii) fluidic components that allow flow of water (and thus also ions) through wicking (i.e. channels in hydrophilic paper defined by wax barriers); iii) electrofluidic components that allow conduction of both ions (in the aqueous medium that fills the pores), and electrons (through the conducting ink that covers the fibers); iv) electrical components that are capable of conducting electrons but not aqueous solutions (comprising fibers coated with an inner layer of electrical conductors, and covered by a second layer of hydrophobic wax). These four components can be patterned in both 2D (one layer of paper), and 3D structures (between layers of stacked paper).

To characterize the basic electrical features of the conductors we measured their sheet resistance to be $70 \Omega /$ sq for PEDOT and $180 \Omega /$ sq for MWNT (see experimental details). The lowest reported sheet resistance values for single-layer planar thin films is $\sim 300 \Omega$ /sq for PEDOT:PSS ${ }^{[25]}$ and $\sim 1000 \Omega /$ sq for polymer dispersed MWNTs. ${ }^{[26]}$ This difference suggests that we utilize the internal surface area of paper for electronic conduction, (i.e., more surface area per 2D printed area), without blocking the pores. This class of electronic structures has four extra properties that differentiate them from conventional wires printed on top of planar substrates: i) They are more conductive than printed carbon- or PEDOT thin films. ii) They penetrate the bulk of the paper and are, therefore, accessible from both sides of the 
paper. iii) They can connect several stacked layers of paper, and thus enable 3D wires without requiring holes in the paper. iv) They do not break easily when folded, and they are protected from other forms of surface damage. To demonstrate some of the capabilities that arise from these four properties we demonstrated two types of printed circuit boards.

In the first demonstration, we designed a three-layer electronic paper circuit that consisted of channels connected through vertical patterns in the middle layer, to form electrical vias. We wax printed the circuit on a single sheet of paper, and then folded and glued the three layers into a single multi-layer device (Figure 2A). PEDOT ink was added in the fluidic channels and connected the paper structure by wicking through all three layers (Figure 2B). After drying of the ink, electrically conducting wires formed both in-plane, through-plane, and between planes, as can be seen from the ability of the wires to power the LEDs in Figure 2B. The possibility of fabricating continuous wires, in one step, through three layers of paper by wicking, make it possible to fabricate multi-layer circuit boards (like the PCBs used commonly in electronics).

In a second demonstration we fabricated a more complicated circuit with several off-the-shelf electronic components (including a microprocessor, transistors, an LEDs), mounted on a paper PCB (see Figure 3A,B and C). We used PEDOT ink to form the conducting wires in the circuit, for making a resistive heating element and resistors. The resolution of the wax patterns enabled wires with a minimum feature size of $1 \mathrm{~mm}$, sufficiently small for the attachment of surface-mounted devices (SMD) . The device was folded in the middle, to form a two-layer structure and to place the resistive heater and the fluidic channel in contact (see Figure 3B). The microprocessor in the device was programmed to detect the presence of a fluid inside the microfluidic 
channel, and execute a programmed thermal cycle (see Figure 3D). The photographs in Figure 3E (left) show the addition of the liquid to the device. This initiated the thermal cycle. The time-lapse infrared images in Figure 3E (right) show the equilibrium temperature for each temperature cycle. The ability to monolithically integrate 3D microfluidics, 3D electrofluidics, and standard microelectronics, enable fabrication of devices that cannot be achieved with traditional PCB manufacturing methods, that are low enough in cost to be single-use and disposable (the total cost of the components in this experiment was 1.3 USD).

The most important and unique properties of the fabrication technique come from the ability of electrofluidic structures to allow simultaneous conduction of both ions, and electrons, and thus act as electrodes in electrochemical paper devices. The ability of liquids to pass through the structure also enables the design of vertical-flow devices (i.e. devices in which electrodes can be stacked using several layers of paper). To analyze the electrochemical behavior of the electrofluidic structure, and use them in devices, we demonstrated two classes of devices: i) electroanalytical devices (Figure 4) and ii) paper-based batteries (Figure 5).

For the electroanalytical device we designed a vertical-flow structure that consisted of three layers of stacked paper (Figure 4A). The device was printed on a single sheet and folded into a three layers (Figure 4B), and consisted of a working-, and a counter-electrode located at the outer layers, and a $\mathrm{Ag} / \mathrm{AgCl}$ pseudo-reference electrode printed in the middle layer. The electrodes extended to wax-coated hydrophobic electrical wires, which were connected to a potentiostat. We added an aqueous electrolyte, (phosphate-buffered saline, 1xPBS, pH 7.0) to the device (Figure 4A - right), and recorded cyclic voltammograms (CVs) for MWNTs and PEDOT electrofluidic electrodes (Figure 4C- top). The CV curves for both the MWNT and the 
PEDOT electrodes were symmetrical with respect to the zero current. This currentvoltage response is typical of supercapacitors with an internal resistance. The shape of the CV shows that the electrofluidic structures do not dissolve or disconnect when filled with water. We calculated the specific capacitance, $C_{s}$ from the CVs to $43 \pm 2 \mathrm{~F} / \mathrm{g}$ for PEDOT and $23 \pm 1 \mathrm{~F} / \mathrm{g}$ for MWNT electrodes (see experimental section). These values are similar to the reported values from the same materials $(\sim 50 \mathrm{~F} / \mathrm{g}$ for PEDOT:PSS ${ }^{[27]}$, and $\sim 25 \mathrm{~F} / \mathrm{g}$ for untreated MWNTs ${ }^{[28]}$ ), which suggest that the majority of the conducting material deposited in the electrofluidic structures is electrically connected and electrochemically active. Since the conducting inks form films around the fibers of the paper, the surface area of the electrodes follows the internal surface area of the paper, rather than the projected, smaller, 2D area.

The high surface area and short diffusion distance inside the electrofluidic structures is favorable for coulometry, which is a technique that has not been fully exploited for paper. In coulometry the charge (calculated from $i$ - $t$ curves) is related to the amount of analyte using Faraday's law. ${ }^{[29]}$ This method can, therefore, allow quantitative electroanalysis without the need for calibration..

We used the vertical-flow device with MWNTs for working and counter electrodes, and used potassium ferrocyanide as our model analyte to perform potentiostatic coulometry. To eliminate the contribution of the double layer, we first added 1xPBS to the device; this addition resulted in an initially high capacitive charging current, which approached a baseline (Figure S2a). Next, we added $2 \mu \mathrm{L}$ of sample solutions of ferrocyanide to the working electrode, in a sequence with increasing concentrations, and measured the area under time-current peak for each sample, corresponding to the total charge $Q$ (see experimental). 
By using Faraday's first law $Q=n N F$ (where $n$ is the number of electrons, $N$ is the moles of electroactive species, and $F$ is Faraday's constant), we plotted $Q / n F$ ( $n=1$ for ferrocyanide) $v s . N$, as seen in Figure 4D. The line represents the ideal straight line $y=x$ (which should be obtained for $100 \%$ conversion efficiency), and the measured data follow this line with a maximum relative error of $18 \%$ between the ideal and the measured data.

Since we could successfully measure the concentration of ferrocyanide using coulometry, we also used this method to fabricate a glucose sensor (see experimental details). We added samples of glucose in 1XPBS (with different concentrations between $2.7-22.2 \mathrm{mM}$, which covers the clinically relevant range in human blood) to the device. The glucose was oxidized to gluconic acid which reduced the cofactor FAD (flavine adenine dinucleotide) to $\mathrm{FADH}_{2} . \mathrm{FADH}_{2}$ was then reoxidized to FAD by ferricyanide, which was converted to ferrocyanide. ${ }^{[30]}$ The chronoamperometric data showed that the production of ferrocyanide required less than 10 seconds (after addition of glucose), to reach a peak current. The ferrocyanide produced was completely converted to ferricyanide in less than 1 minute (see Figure S2). We estimate that wicking into the electrodes, diffusion inside the electrodes, and full enzymatic conversion, all can occur in less than a second (see supporting Information). These should therefore not be rate limiting. Instead, a combination of the diffusion and mixing in the paper, and the internal resistance of the electrodes (due to potential drop at high currents) could be the main factors that affect the speed of the measurement.

We used the same procedure as described previously to measure the amount of glucose $v s$. the added amount of glucose, and plotted the data in Figure 4E. The line in Figure 4E displays the linear ratio $(\mathrm{y}=\mathrm{x})$ expected for ideal behavior. The measured 
values follow this line, and the concentration of glucose can be directly estimated without calibration. Considering the amount of added glucose as the true value, the maximum error was $19 \%$, which indicates that the methodology is sufficiently accurate for clinical use. Because it is absolute, it could (with further engineering improvement) be used to assess the accuracy of glucose monitors.

To summarize, vertical-flow electroanalytical devices can be fabricated using electrofluidic structures, and that their high surface area, and short diffusion distances, enables rapid and complete conversion of analytes. This new capability has the potential to enable quantitative electroanalysis without the need for calibration; a calibration-free method can decrease time, cost, and error.

The combination of electrochemical stability, high specific capacitance, high surface area, and three-dimensional configurability of electrofluidic structures is also an advantage for energy-storage devices. To demonstrate energy-storage, we fabricated paper batteries, by printing the entire battery on a single sheet of paper, where the electrofluidic as well as the electronic structures of the battery were fabricated using PEDOT. The printed single sheet could be folded into a final battery structure (Figure 5A). In this particular design, we integrated six batteries, connected in series, through the paper conductors to achieve higher voltages. In addition, folding aligns the patterns in each layer. Since PEDOT can have different reduction states, it can act both as anode or cathode in a battery. We demonstrated a battery, which is charged in its initial dry state. The charging was achieved by adding the polymer polyethylenimine (PEI), to the conducting, PEDOT-infused paper. PEI reduced PEDOT, so that it acted as an anode. The reduced PEDOT has a darker blue color than pristine PEDOT (Figure 5B). This type of battery has been described before as a thin film device. ${ }^{[31]}$ These paper batteries can be stored in dry form, and activated 
when an electrolyte is added. We connected the batteries to a red LED, and generated a simple electrical push-button by folding the edge of the paper (see Figure 5D, 5E). We added $1 \mathrm{M}$ aqueous $\mathrm{NaCl}$ solution to activate the paper battery, and then pushed the button to turn on the LED (Figure 5D). The LED remained on for 15 seconds before the voltage dropped below $\sim 2 \mathrm{~V}$. The initial voltage was $2.5 \mathrm{~V}$ corresponding to the additive voltage of each individual battery element (around $0.5 \mathrm{~V}$ ). A full discharge curve of the battery at a constant current of $5 \mu \mathrm{A}$ showed a decreasing voltage from $2.5 \mathrm{~V}$ down to $0 \mathrm{~V}$ over the course of around one hour (see Figure S3), with an energy of $\sim 6 \mu \mathrm{Wh}$, corresponding to a specific energy of $833 \mathrm{Wh} / \mathrm{kg}$ (when we account only for the weight of PEDOT), or $26 \mathrm{Wh} / \mathrm{kg}(94 \mathrm{~kJ} / \mathrm{kg})$ accounting for total weight of the paper batteries (see experimental). For comparison, the specific energy of rechargeable lithium ion batteries is around $400 \mathrm{~kJ} / \mathrm{kg}$. These paper batteries are light, can be stored in dry form, and can be integrated monolithically with the other paper devices.

In conclusion, this paper demonstrates a step toward the full integration of electronic and microfluidic functions in paper devices by exploiting the ability of hydrophobic patterns printed in paper to define a new class of electrical conductors. These conductors are "electrofluidic", in the sense that a single channel can act as wires, electrodes, and microfluidic channels at the same time. Patterned electrofluidic structures provide five new capabilities to paper devices: i) They have a high surface area (and a small diffusion time for electrochemical reactions) as they utilize the internal surface of paper rather than just the projected printed area. ii) They allow flow of both electrons and fluids, and thus integrate paper microfluidics and paper electronics monolithically. iii) They allow the printing of electrical wires with low resistance. iv) They enable fabrication of complex patterns of electronic and 
electrochemical structures that can be connected to each other through several layers of paper (i.e. connected in 3D). v) They are resistant to creasing or surface damage, and can, therefore, be used in configurable paper devices that require folding.

Using these new capabilities, we have demonstrated the fabrication of 3D printed circuit boards (PCBs) that allow mounting of conventional circuit elements (such as microprocessors). These paper PCBs extend the function of conventional PCBs by adding microfluidic and reconfiguration capabilities to circuits. We have shown foldable, fully printed, and all-organic paper batteries with (non-optimized) specific energy densities of $\sim 6 \mu \mathrm{Wh}(26 \mathrm{Wh} / \mathrm{kg})$. We have also demonstrated electroanalytical paper devices that carry out coulometry, which allows quantitative analysis without calibration.

The method we describe should not be limited to the specific conducting inks we have used. Wicking inside microfluidic channels could be used to pattern many other types of functional materials from suspensions in water, including metallic, ${ }^{[32]}$ semiconducting, ${ }^{[33,34]}$ and magnetic nanostructures, or optical polymers. We believe that these methods for integrating electronics and microfluidics, by printing on paper, enables a number of new designs and capabilities for printed paper devices. It will be especially interesting to explore in applications where bulk electrochemical conversion (hence, requiring large surface area), and/or complex 3D electronics and electrofluidic micro patterns are important. Examples might include paper-based batteries, ${ }^{[35,36]}$ fuel cells, ${ }^{[37,38]}$ electroanalytical devices, ${ }^{[5]}$ dye-sensitized solar cells, ${ }^{[39-41]}$ light-emitting electrochemical cells, ${ }^{[42]}$ electrochemical transistors, ${ }^{[43,44]}$ electrochromic displays, ${ }^{[45]}$ electrochemical actuators for drug release,${ }^{[46]}$ or microrobots. ${ }^{[47]}$ 
Figures

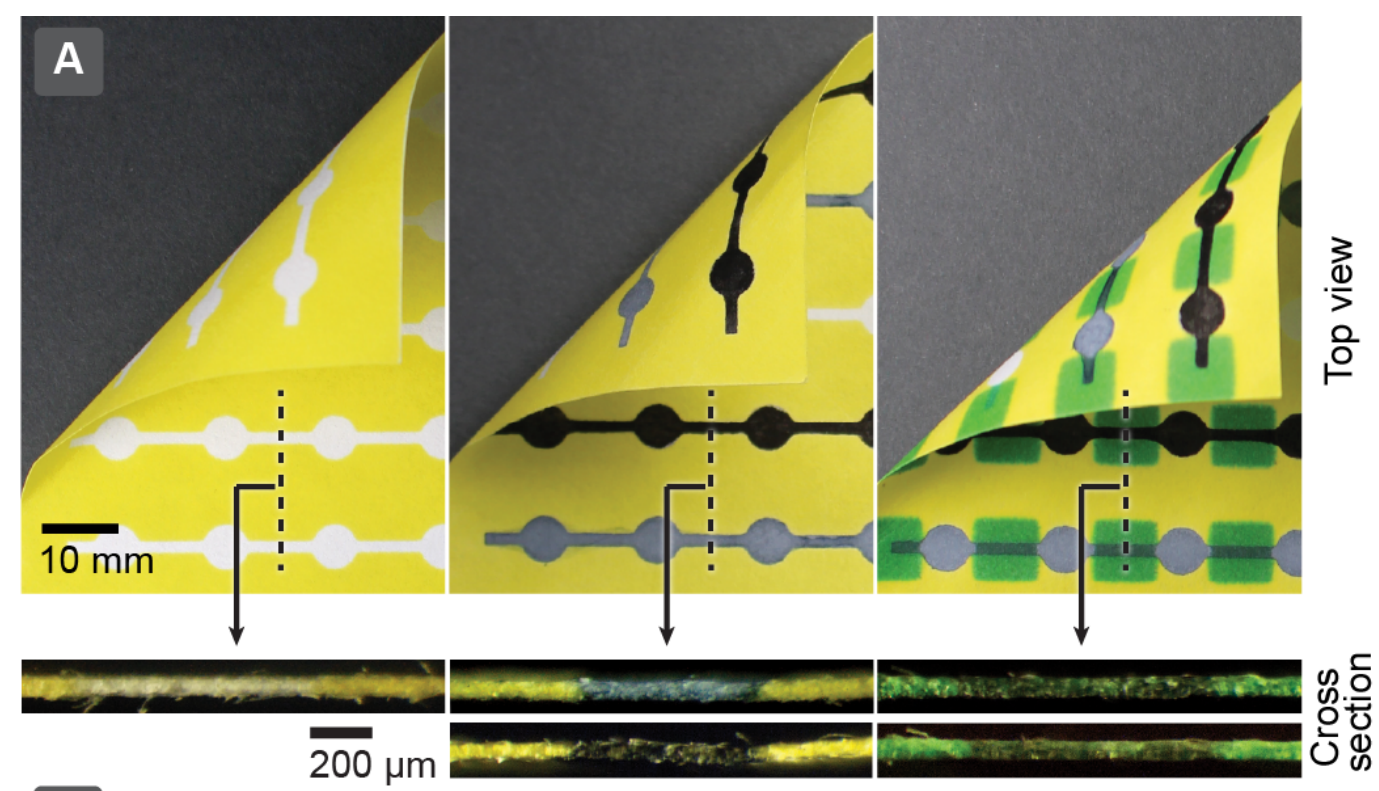

B i)

ii)

iii)

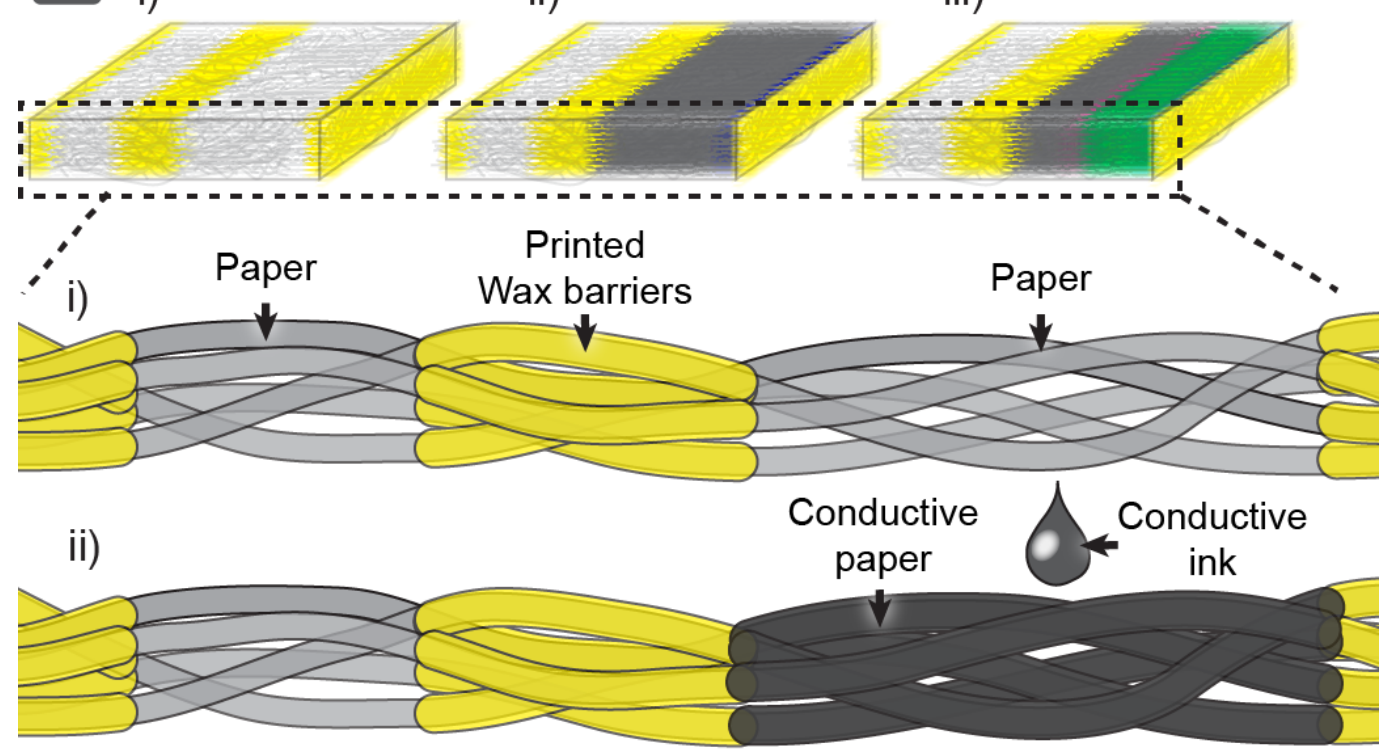

Printed

iii)

Wax barriers
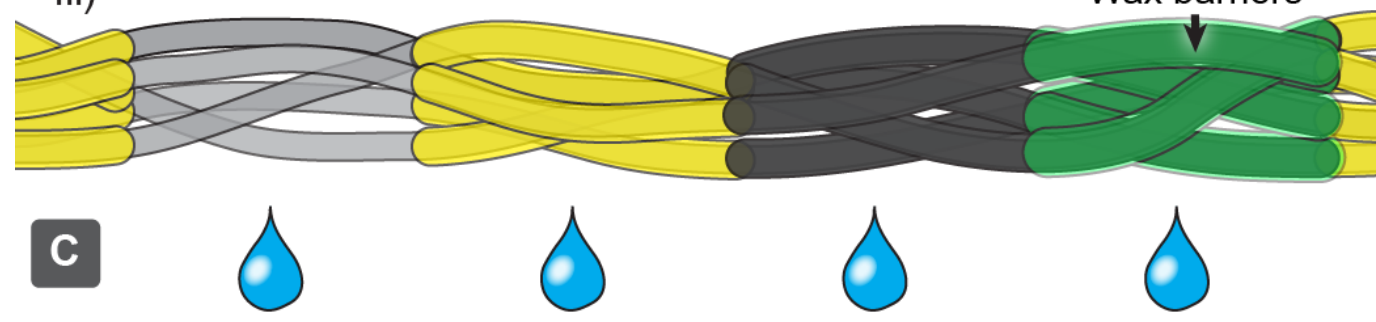

1

0

0

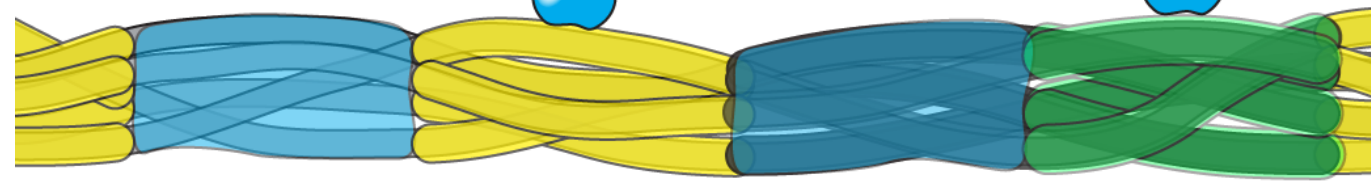

Fluidic channel

Barrier

Electrofluidic channel

Electric wire 
Figure 1. Co-fabrication of printed electronics and printed microfluidics. A) Top view photos (top) of the printed structures, in three steps according to the schematics in figure 1B (yellow ink is wax, blue ink is PEDOT and black ink is MWNTs). (bottom) Cross section photos of the paper in each step B) Schematic diagram of the fabrication procedure: i) Wax is printed to form microfluidic structures in paper. ii) water-based conductive inks are added to the parts of the microfluidic structures to pattern electrofluidic structures, iii) a second layer of wax is printed to make parts of the electrofluidic structures hydrophobic, thereby forming electric wires. C) Schematic diagram of the cross section of a printed structure filled with an aqueous electrolyte, whereupon fluidic, or electrofluidic structures are formed. The electrolyte does not wick the hydrophobic wax printed structures (barrier and electric wire). 

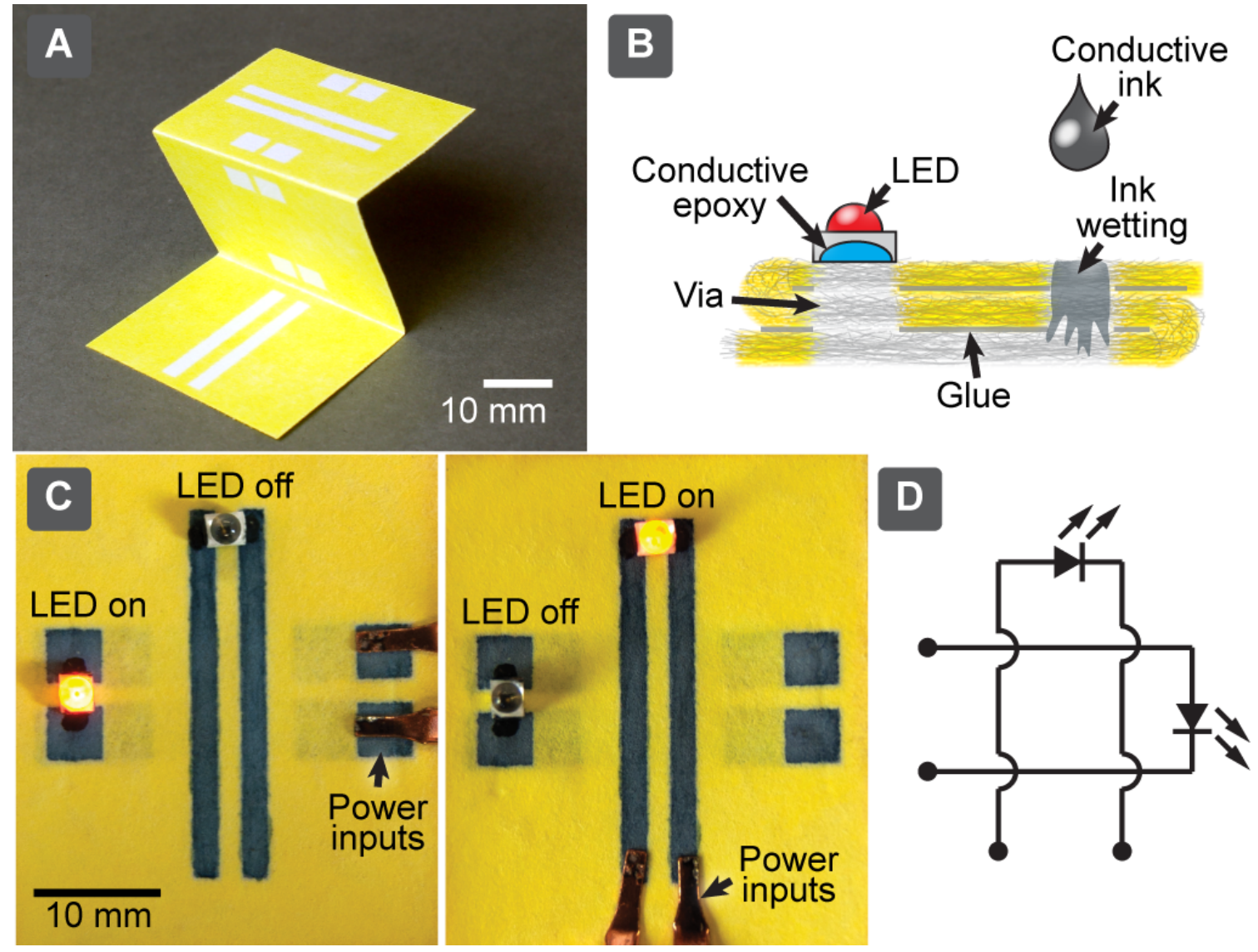

Figure 2. Printed multi-layer circuit board on paper A) Photo of a wax printed channels, for forming a 3D circuit board, before folding. B) Schematic diagram of the fabrication of the 3D circuit, where the wax printed paper, has been folded and glued into a three-layer structure. PEDOT was then added to the channels to form 3D electrical wires (through spontaneous wicking), and Light Emitting Diodes, (LEDs), are mounted on top (using conducting glue). C) Top view photos of the circuit. (left) The LED is powered through the two lines in the bottom layer connected to the top layer through vias and, (right) The LED is powered through the two top lines. D) Circuit diagram of the device. 


\section{A}
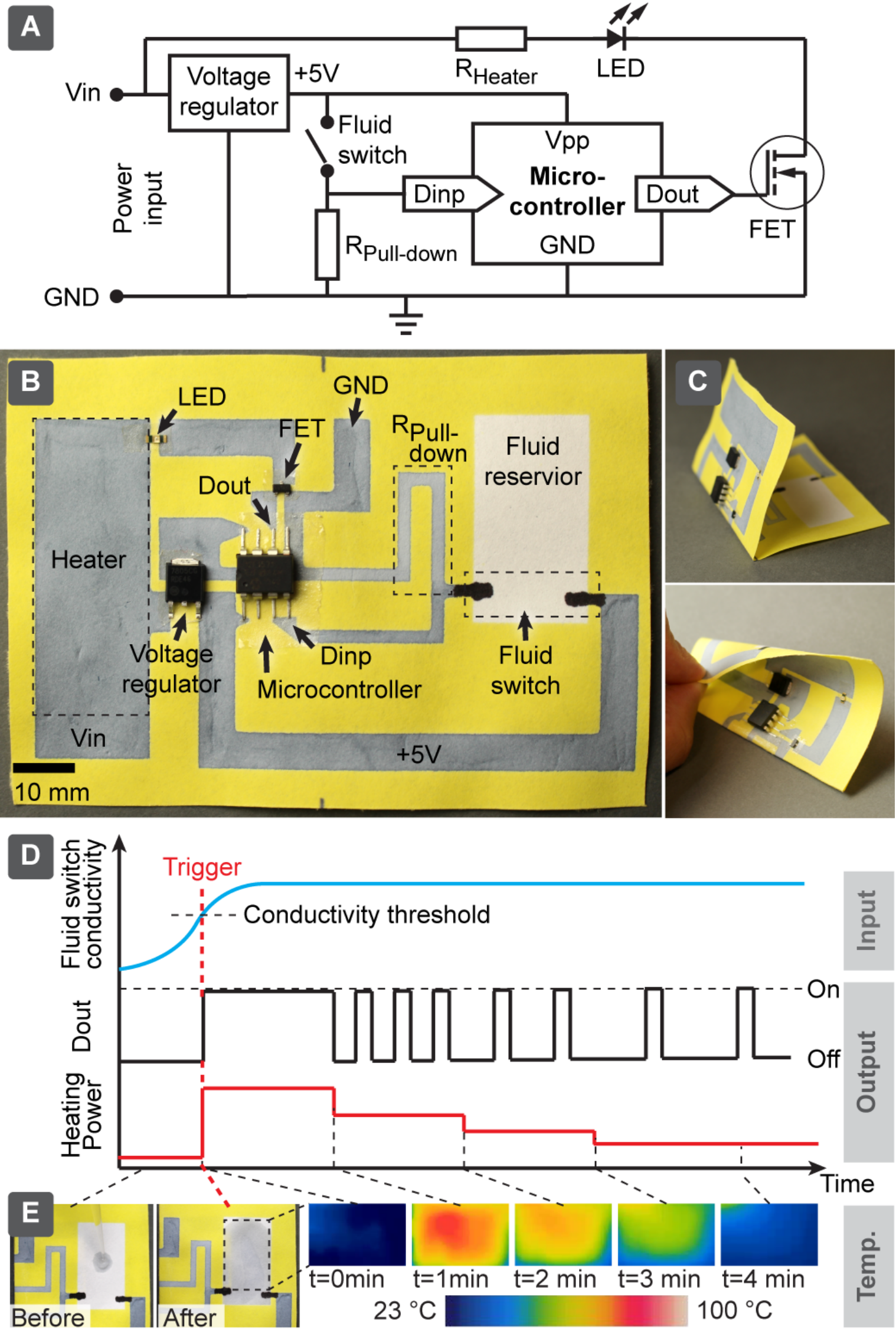
Figure 3. Printed circuit boards: a microcontroller based heater. A) Circuit diagram for a microcontroller based heating device. B) Photo of the device described by the diagram in A, where all the wires are printed in paper, using wax printing and PEDOT inks. The electrical components are mounted on one side using electrically conductive adhesive tapes. C) Photos showing (left) folding of the paper circuit to form the final device (right) with all the components on one side and the fluidic reservoir on the other side. D) A schematic diagram showing the analog input, and digital output of the microcontroller as well as the measured heating power, as a function of time. E) (left) Photos of showing water (with $100 \mathrm{mM} \mathrm{KCl}$ ) placed in the fluid reservoir with a pipette. When the water reached the fluid detector the microcontroller started a heating cycle according to the schematic figure in $3 \mathrm{D}$. The heat $v s$. time of the wet paper is seen in the IR time-lapse photos in Figure 3E left. 


\section{A}

$$
\begin{aligned}
& \text { Working } \\
& \text { electrode (WE) }
\end{aligned}
$$

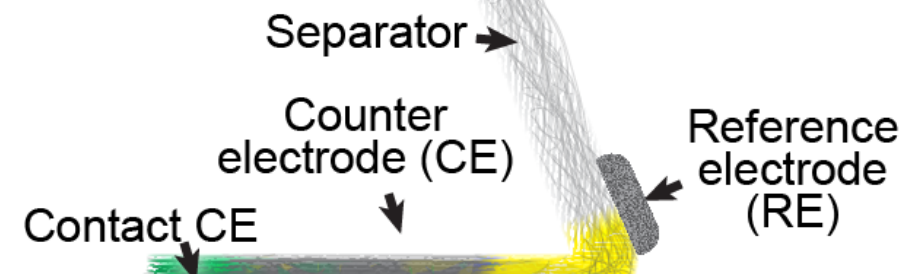

B

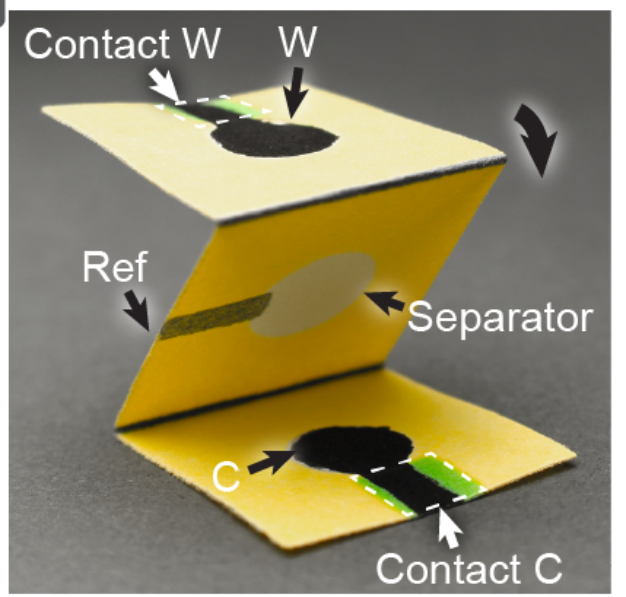

D

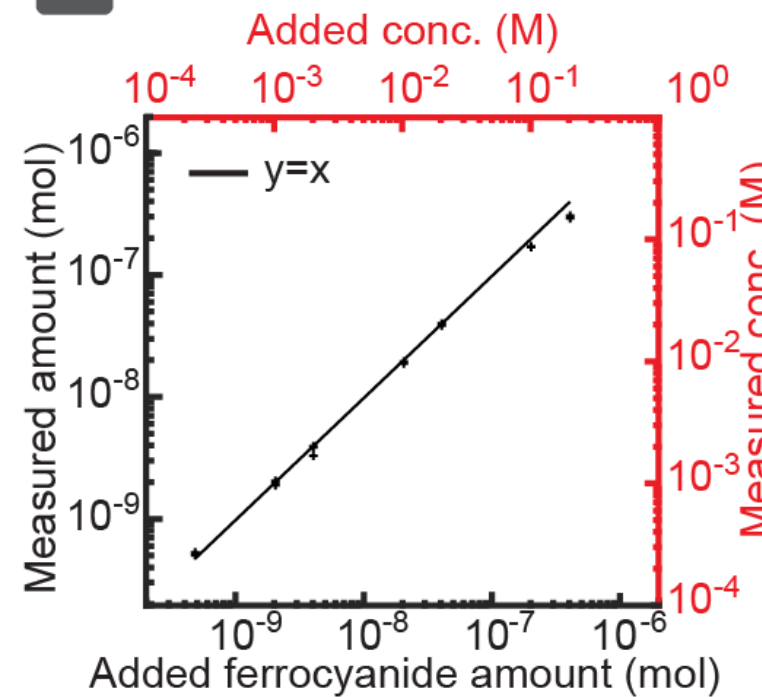

C

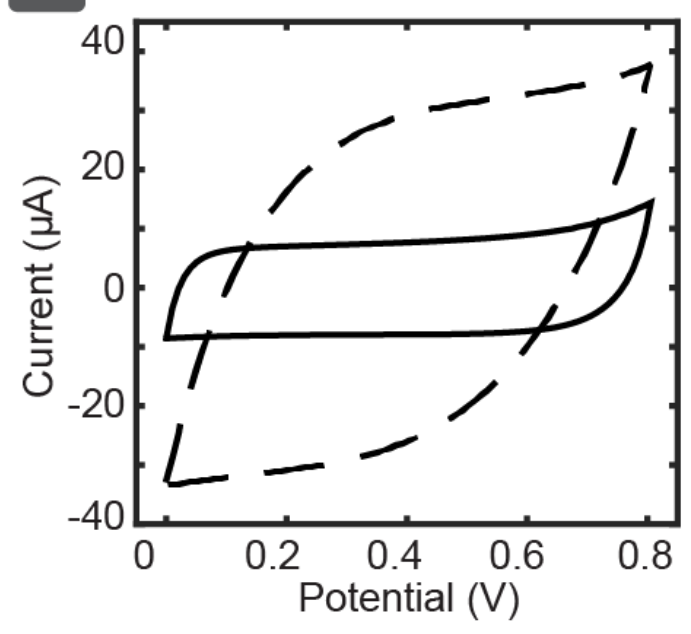

E

Sample
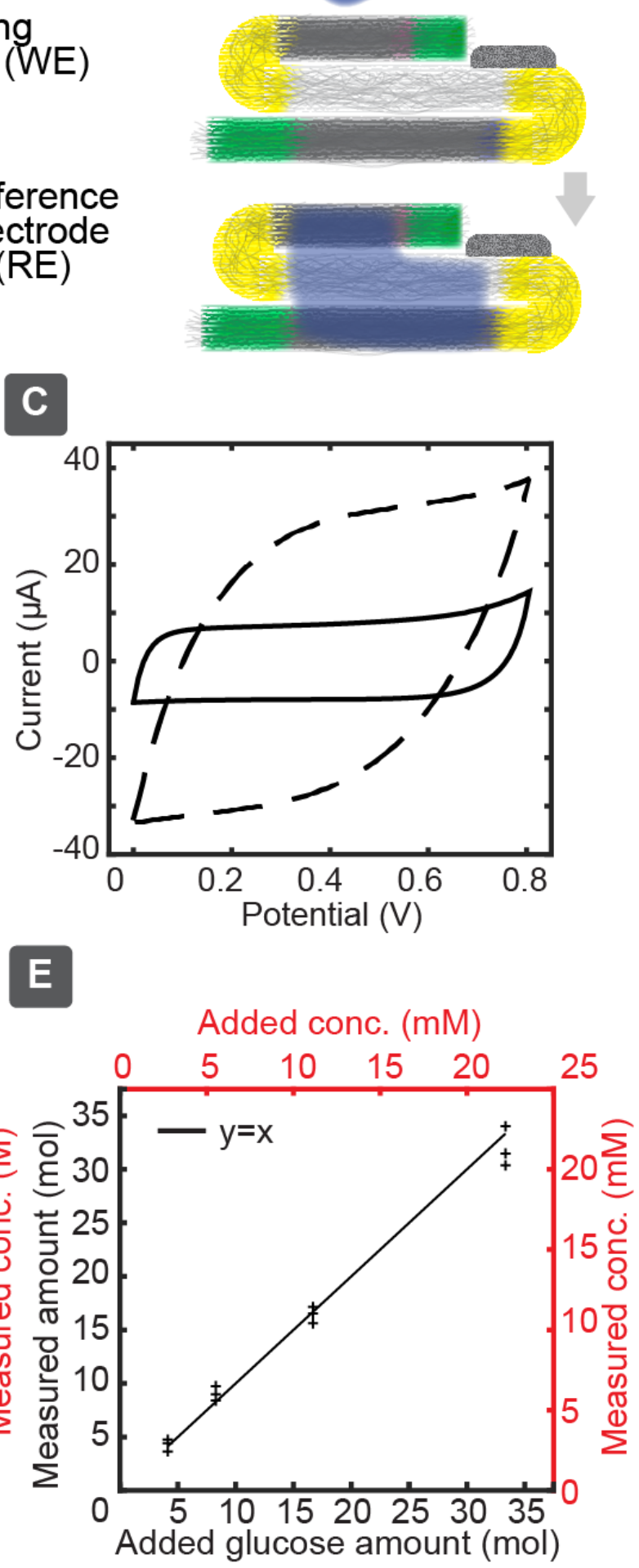
Figure 4. Vertical-flow electroanalytical paper devices A) Schematic side view diagram of the assembly and operation of an electroanalytical device. The device is first printed on a single piece of paper using wax printing and conducting inks to form electrofluidic working-and counter-electrodes, and electrical contact points. A $\mathrm{Ag} / \mathrm{AgCl}$ ink is printed in the middle layer as a reference electrode. The paper is then folded (left) to form the device (right). The device works by adding a sample on the working electrode; The fluid containing the sample then wicks through the electrodes, and forms an electrochemical circuit. B) Photo of the device before folding into the final structure $\mathbf{C}$ ) Cyclic voltammograms (CVs) recorded in phosphate-buffered saline (1xPBS, pH 7.0) on PEDOT electrodes (dashed), and MWNT electrodes (solid), at $10 \mathrm{mV} / \mathrm{s}$. D) Results obtained by potentiostatic (+0.6 V) coulometry for ferrocyanide in 1xPBS $(\mathrm{n}=4)$. The measured amount of ferrocyanide in moles, $N$, was calculated from the coulometric charge $Q$ using $N=Q / F$, and represented $v s$. the added amount. The line shows the 1:1 linear relation $(\mathrm{y}=\mathrm{x})$. E) Results obtained by potentiostatic coulometry $(+0.6 \mathrm{~V})$ for a glucose assay $(\mathrm{n}=3)$. The measured amount of glucose in moles, $N$, was calculated from the charge $Q$ using $N=Q / 2 F$. (Two ferrocyanide ions are produced from ferricyanide for each glucose) and represented vs. the added amount. The line shows the 1:1 linear relation $(\mathrm{y}=\mathrm{x})$. In both $\mathrm{D})$, and $\mathrm{E})$ the concentration is calculated as (calculated amount/added volume). 

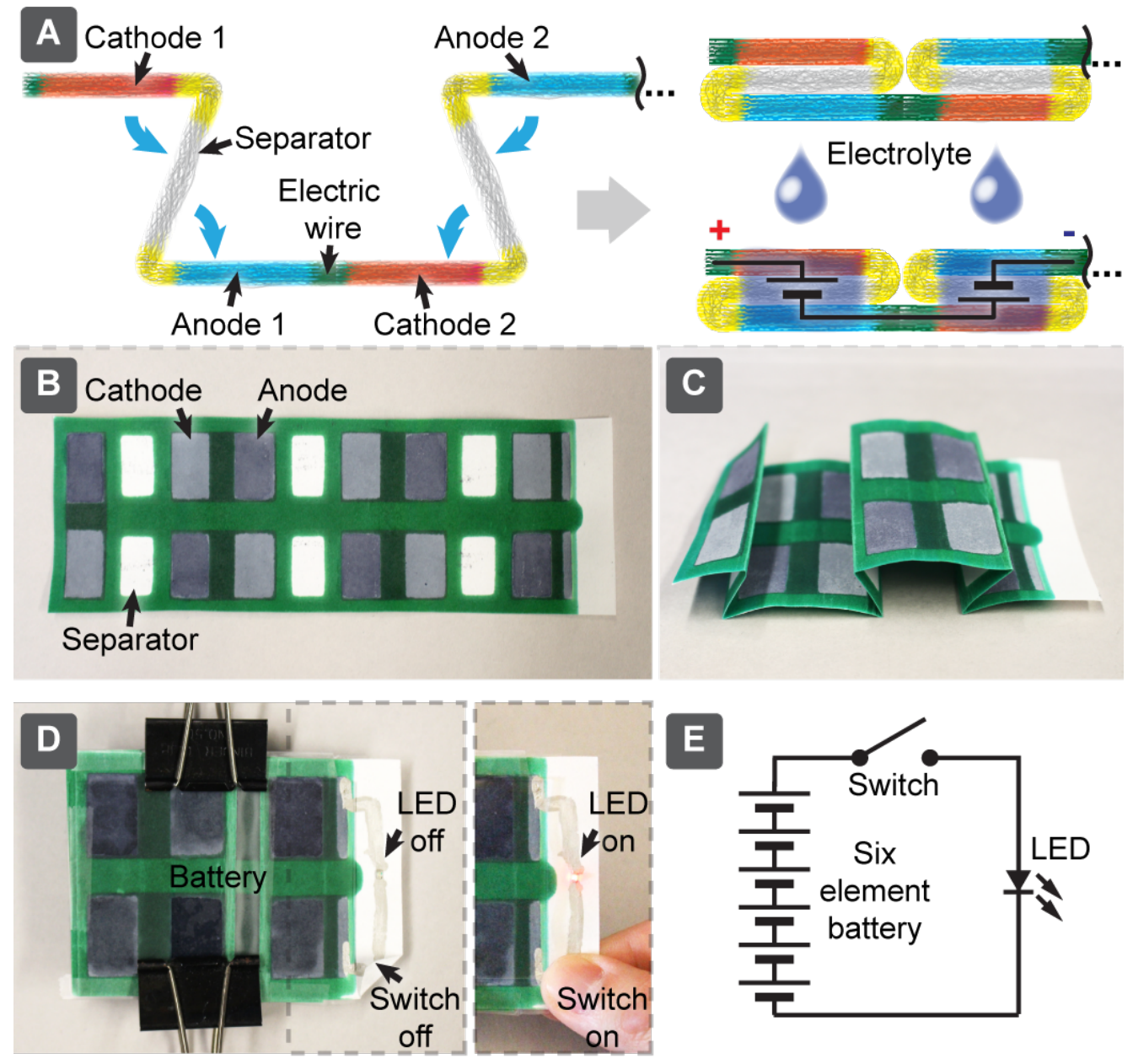

Figure 5. Foldable paper batteries. A) Schematics diagram of the assembly of a printed paper battery: The battery is fabricated on a single paper using wax printing and printing of PEDOT to form electrofluidic structures (andoes and cathodes) and electric wires. The paper is then folded according to Figure 5C and 5A to form the final battery structure, and is activated by adding electrolyte to the anodes or cathodes. B) Photo of a fully printed device with six serial connected batteries before folding, and C) during folding D) Photo of the final folded battery with electrolyte (1 $\mathrm{M} \mathrm{NaCl}$ ) added. The batteries were connected with painted electrical wires, to an LED that was mounted on the paper using conductive glue. The LED was controlled with a paper switch button. The switch is in the off-state on the left photo, and in the on-state on the right photo. When switched on the batteries initially provided a 
voltage of 2.5 Volt at $12.5 \mu \mathrm{W}$, and the LED emitted light for 15 seconds. E) Equivalent electrical circuit of the device in D).

\section{Experimental Section}

Materials: We purchased poly(3,4-

ethylenedioxythiophene):poly(styrenesulfonic acid) Clevios ${ }^{\mathrm{TM}} \mathrm{PH} 1000$ from Heraeus. Multiwall carbon nanotubes MWNTs ( $>95 \%$ carbon, product of Southwest Nanotechnology), branched polyethylenimine PEI (60 kDa), potassium ferrocyanide, potassium ferricyanide, Glucose oxidase, and Glucose, were purchased from Sigma Aldrich. 3-Aminopropoyldimethylethoxysilane was purchased from Gelest Inc. Carboxymethyl cellulose, sodium salt (M.W. 250 000), and 3-glycidoxypropyltrimethoxysilane (97\%), where purchased from Acros Organics.

Preparation of Organic Water-Based Conducting Ink: The conducting polymer (PEDOT:PSS) ink (10 mg/mL dispersed in water) was mixed with $5 \mathrm{wt} \%$ dimethylsulfoxide (DMSO). The addition of DMSO leads to a higher conductivity upon drying because it leads to more phase separation between PEDOT and PSS ${ }^{[48]}$. To make the carbon ink, MWNTs powder ( $5 \mathrm{~g} / \mathrm{L})$ was mixed with dissolved carboxymethyl cellulose in de-ionized water $(2.5 \mathrm{~g} / \mathrm{L})$. The mixture was ultrasonicated with a tapered micro-tip for 15 min using a Branson sonifier 340 with an output power of $400 \mathrm{~W}$. The sonicated ink was purified by centrifuging at 4500 rpm for $30 \mathrm{~min}$, and then decanting.

Printing Electrofluidic Devices: A first layer of wax was printed with a Xerox ColorQube Wax printer on Whatman ${ }^{\circledR}$ cellulose chromatography paper (grade $1 \mathrm{Chr}$ ). The wax was subsequently melted into the paper by heating the paper in an oven at $140{ }^{\circ} \mathrm{C}$ for 90 seconds. The conducting inks were added with a pipette into the paper 
channels defined by the wax. The ink was allowed to dry through evaporation at room temperature, or in an oven at $70{ }^{\circ} \mathrm{C}$. A second layer of wax was printed onto the structures and again melted using the same procedure as above, to create a pattern of purely electronic structures. To increase the fluidic properties and spontaneous wicking of the conducting parts, we treated some of the conducting parts with silane solutions, after the conducting inks had dried. We added a $2 \mathrm{wt} \%$ solution of 3glycidoxypropyl-trimethoxysilane to the PEDOT:PSS structures, and we added $2 \mathrm{wt} \%$ solution of 3-aminopropoyldimethylethoxysilane (in deionized water) to the MWNT structures.

Electrical characterization: We used $30 \mu \mathrm{L} / \mathrm{cm}^{2}$ of PEDOT $(10 \mathrm{mg} / \mathrm{mL})$ and MWNTs $(5 \mathrm{mg} / \mathrm{mL})$ water-based inks to pattern conducting lines (with widths of 1-4 $\mathrm{mm}$, the thickness of the paper was $180 \mu \mathrm{m})$, and measured the resistance using two point probe techniques. To test folding cycles, we fabricated a conducting line $(1 \mathrm{~mm}$ by $18 \mathrm{~cm}$ ), and made an accordion fold with 11 zig-zag folds along the conductor. We performed folding cycles, from unfolded to fully folded (180 degrees), and measured the resistance along the wire as a function of the fold angle.

Calculations of Capacitance: The calculation of the specific capacitance for the electrofluidic structures was done by recording CVs using a two-electrode device (electrodes located at the outer layers of the three-layer device). The electrofluidic structures were patterned using $15 \mu \mathrm{L}$ of PEDOT ink $(10 \mathrm{mg} / \mathrm{mL}$, corresponding to a total mass of $150 \mu \mathrm{g}$ ), and $15 \mu \mathrm{L}$ of MWNTs inks ( $5 \mathrm{mg} / \mathrm{mL}$, corresponding to a mass of $75 \mu \mathrm{g}$ ). 1xPBS (pH 7.0) was used as electrolyte. CVs were recorded at sweep rates, between 5 and $100 \mathrm{mV} / \mathrm{s}$, using an Autolab potentiostat ( $\mathrm{N}$ series) from Metrohm. The specific capacitance $\mathrm{C}_{\mathrm{s}}$ was calculated using equation $C s=2 i m d E d t$, where $i$ is the current measured in the electrolyte solution at $0.5 \mathrm{~V}(v s . \mathrm{Ag} / \mathrm{AgCl}), d E / d t$ is the sweep 
rate, and $m$ is the total mass of the conductive material. We calculated the capacitance at different sweep-rates and for different devices to obtain an average value.

Fabrication of Electronic Paper Circuits: The circuit board was designed in Adobe $^{\mathrm{TM}}$ Illustrator and printed as above. PEDOT was used in $10 \mathrm{mg} / \mathrm{mL}$ concentration to define the electrically conducting lines with low resistance, and the lines with higher resistance (resistor components) were fabricated using diluted PEDOT (2 mg/mL). Electronic components (microcontroller PIC 12F1571, voltage regulator MC7805, and a MOSFET N-CH transistor The AO3162, LED reverse MNT SMD) were purchased from Digikey Inc. The total price of the components was around 1.3 USD. The components were mounted on the printed circuit board using double-sided electrically conductive adhesive transfer tape from $3 \mathrm{M}^{\mathrm{TM}}$, or using conductive epoxy glue CW200 from Circuitworks ${ }^{\circledR}$. The microprocessor was programmed using MPLAB X IDE from Microchip. After mounting the components, the device was folded to place the fluid channel in contact with the heater. The temperature of the heater was controlled digitally by programming different ratios between the on and off state on the microcontroller. The on-state was kept at a constant of 0.5 seconds, and the off state varied between 0 (maximum heat), to 16 (minimum heat). The paper heater was filmed using an FLIR 600 infrared imaging camera.

Coulometric Measurements for Ferrocyanide: The procedure used in these experiments was as follows:1) We filled the vertical flow three-electrode device with $30 \mu \mathrm{L}$ of a loading electrolyte (1xPBS pH 7). 2) We applied a potential of $0.6 \mathrm{~V} v s$. $\mathrm{Ag} / \mathrm{AgCl}$ to the working electrode and recorded the current vs. time, using an Autolab potentiostat. This procedure generated a relatively large double-layer capacitive current, probably due to the high surface area of the electrodes. 3) After the current 
approached a baseline, small amounts $(2.0 \mu \mathrm{L})$ of a solution of ferrocyanide were added to the working electrode, using a micropipette, in a sequence with increasing concentrations.

Glucose Assay: A volume of $30 \mu \mathrm{L}$ of a solution of $250 \mathrm{U} / \mathrm{mL}$ Glucose oxidase in 1xPBS buffer $\mathrm{pH} 7.0,1 \mathrm{M} \mathrm{KCl}$ and $500 \mathrm{mM}$ Ferricyanide was added to the electrode, and a constant potential of $0.6 \mathrm{~V}$ ( $v s$. the $\mathrm{Ag} / \mathrm{AgCl}$ reference) was applied to the working electrode and the current was recorded vs. time, using an Autolab potentiostat. After 10 min of equilibration time (during which the electrodes were sealed with a tape prevent evaporation), $1.5 \mu \mathrm{L}$ of glucose solution was added on the top of the working electrode for each sample. The final data were analyzed by calculating the area under each peak (corresponding to the charge) with MATLAB ${ }^{\circledR}$. Paper Battery Construction and Characterization: The paper battery was built using printing procedures already described. The PEDOT anodes were made by adding a few drops of $50 \mathrm{mg} / \mathrm{mL}$ of PEI to PEDOT pattern, followed by drying at room temperature. The electrical characterization was made using a Keithley 2400 measurement unit. The specific energy was calculated from these values: a basis weight of Whatman paper of $92 \mathrm{~g} / \mathrm{m}^{2}$, a total area of batteries of $24 \mathrm{~cm}^{2}$ corresponding to a total weight of $220 \mathrm{mg}$ for the paper, and the total weight of PEDOT in the battery of $2.7 \mathrm{mg}$.

\section{Supplementary information}

Supporting Information is available from the Wiley Online Library.

\section{Acknowledgements}

M. M. H. acknowledges support from Marie Curie IOF FP7 for project nanoPAD (Grant Agreement Number 330017), the Bo Rydins stiftelse (SCA AB), and the 
Sweden-America Foundation. F.G. thanks the German Research Foundation (GU

1468/1-1) for research support. D.C.C acknowledges support from the Defense Threat

Reduction Agency, award \#HDTRA1-14-C-0037. M.T.F.A. thanks the University of

Oviedo through the Campus de Excelencia Internacional for partially funding her stay

at Harvard University. A. A. thanks the Swedish Research Council (VR) for a

postdoctoral fellowship.

\section{References}

[1] P. N. Nge, C. I. Rogers, A. T. Woolley, Chem. Rev. 2013, 4, 2550.

[2] E. J. Maxwell, A. D. Mazzeo, G. M. Whitesides, MRS Bull. 2013, 38, 309.

[3] D. G. Rackus, M. H. Shamsi, A. R. Wheeler, Chem. Soc. Rev. 2015, 44, 5320.

[4] W. Dungchai, O. Chailapakul, C. S. Henry, Anal. Chem. 2009, 81, 5821.

[5] D. M. Cate, J. A. Adkins, J. Mettakoonpitak, C. S. Henry, Anal. Chem. 2015, 87, 19.

[6] A. C. Glavan, R. V. Martinez, A. B. Subramaniam, H. J. Yoon, R. M. D. Nunes, H. Lange, M. M. Thuo, G. M. Whitesides, Adv. Funct. Mater. 2014, 24, 60.

[7] A. C. Glavan, D. C. Christodouleas, B. Mosadegh, H. D. Yu, B. S. Smith, J. Lessing, M. T. Ferna, G. M. Whitesides, Anal. Chem. 2014, 86, 11999.

[8] J. Lessing, A. C. Glavan, S. B. Walker, C. Keplinger, J. a Lewis, G. M. Whitesides, Adv. Mater. 2014, 26, 4677.

[9] Z. Nie, C. a Nijhuis, J. Gong, X. Chen, A. Kumachev, A. W. Martinez, M. Narovlyansky, G. M. Whitesides, Lab Chip 2010, 10, 477.

[10] M. Zhang, L. Ge, S. Ge, M. Yan, J. Yu, J. Huang, S. Liu, Biosens. Bioelectron. 2013, 41, 544 .

[11] C. Renault, M. J. Anderson, R. M. Crooks, J. Am. Chem. Soc. 2014, 136, 4616.

[12] C. Renault, X. Li, S. E. Fosdick, R. M. Crooks, Anal. Chem. 2013, 85, 7976.

[13] L. Ge, S. Wang, J. Yu, N. Li, S. Ge, M. Yan, Adv. Funct. Mater. 2013, 23, 3115.

[14] A. K. Yetisen, M. S. Akram, C. R. Lowe, Lab Chip 2013, 13, 2210.

[15] A. W. Martinez, S. T. Phillips, G. M. Whitesides, Anal. Chem. 2010, 82, 3.

[16] E. Carrilho, A. W. Martinez, G. M. Whitesides, Anal. Chem. 2009, 81, 7091.

[17] D. Tobjörk, R. Österbacka, Adv. Mater. 2011, 23, 1935.

[18] M. Berggren, D. Nilsson, N. D. Robinson, Nat. Mater. 2007, 6, 3.

[19] S. R. Forrest, Nature 2004, 428, 911.

[20] W. J. Hyun, E. B. Secor, G. a. Rojas, M. C. Hersam, L. F. Francis, C. D. Frisbie, Adv. Mater. 2015, 27, 7058.

[21] O. Bubnova, Z. U. Khan, H. Wang, S. Braun, D. R. Evans, M. Fabretto, P. Hojati-Talemi, D. Dagnelund, J.-B. Arlin, Y. H. Geerts, S. Desbief, D. W. Breiby, J. W. Andreasen, R. Lazzaroni, W. M. Chen, I. Zozoulenko, M. Fahlman, P. J. Murphy, M. Berggren, X. Crispin, Nat. Mater. 2014, 13, 190.

[22] M. Wagner, G. Lisak, A. Ivaska, J. Bobacka, Sensors Actuators B Chem. 2013, 181,694 . 
[23] M. Pumera, Chem. Eur. J. 2009, 15, 4970.

[24] N. Minami, Y. Kim, K. Miyashita, S. Kazaoui, B. Nalini, Appl. Phys. Lett. 2006, 88, 093123.

[25] M. Vosgueritchian, D. J. Lipomi, Z. Bao, Adv. Funct. Mater. 2012, 22, 421.

[26] V. Bocharova, A. Kiriy, U. Oertel, M. Stamm, F. Stoffelbach, R. Jérôme, C. Detrembleur, J. Phys. Chem. B 2006, 110, 14640.

[27] X. Ma, W. Zhou, Z. Wang, D. Mo, X. Duan, J. Xu, J. Solid State Electrochem. 2015, 52, 1989.

[28] S. Shiraishi, M. Kibe, T. Yokoyama, H. Kurihara, N. Patel, A. Oya, Y. Kaburagi, Y. Hishiyama, Appl. Phys. A Mater. Sci. Process. 2006, 82, 585.

[29] J. Lankelma, Z. Nie, E. Carrilho, G. M. Whitesides, Anal. Chem. 2012, 84, 4147.

[30] A. Heller, B. Feldman, Chem. Rev. 2008, 108, 2482.

[31] Y. Xuan, M. Sandberg, M. Berggren, X. Crispin, Org. Electron. 2012, 13, 632.

[32] Z. Chen, A. R. Rathmell, S. Ye, A. R. Wilson, B. J. Wiley, Angew. Chem. Int. Ed. Engl. 2013, 52, 13708.

[33] L.-F. Cui, R. Ruffo, C. K. Chan, H. Peng, Y. Cui, Nano Lett. 2009, 9, 491.

[34] L. Wang, T. Sasaki, Chem. Rev. 2014, 114, 9455.

[35] L. Nyholm, G. Nyström, A. Mihranyan, M. Strømme, Adv. Mater. 2011, 23, 3751.

[36] L. Hu, J. W. Choi, Y. Yang, S. Jeong, F. La Mantia, L.-F. Cui, Y. Cui, Proc. Natl. Acad. Sci. 2009, 106, 1.

[37] N. K. Thom, K. Yeung, M. B. Pillion, S. T. Phillips, Lab Chip 2012, 12, 1768.

[38] J. P. Esquivel, F. J. Del Campo, J. L. Gómez de la Fuente, S. Rojas, N. Sabaté, Energy Environ. Sci. 2014, 7, 1744.

[39] E. C. P. Smits, S. G. J. Mathijssen, P. a. van Hal, S. Setayesh, T. C. T. Geuns, K. a. H. a. Mutsaers, E. Cantatore, H. J. Wondergem, O. Werzer, R. Resel, M. Kemerink, S. Kirchmeyer, A. M. Muzafarov, S. a. Ponomarenko, B. de Boer, P. W. M. Blom, D. M. de Leeuw, Nature 2008, 455, 956.

[40] B. Wang, L. L. Kerr, Sol. Energy Mater. Sol. Cells 2011, 95, 2531.

[41] M. J. Yun, S. I. Cha, S. H. Seo, H. S. Kim, D. Y. Lee, Sci. Rep. 2015, 5:11022.

[42] A. Asadpoordarvish, A. Sandström, C. Larsen, R. Bollström, M. Toivakka, R. Österbacka, L. Edman, Adv. Funct. Mater. 2015, 25, 3238.

[43] M. Sessolo, J. Rivnay, E. Bandiello, G. G. Malliaras, H. J. Bolink, Adv. Mater. 2014, 26, 4803.

[44] D. Khodagholy, J. Rivnay, M. Sessolo, M. Gurfinkel, P. Leleux, L. H. Jimison, E. Stavrinidou, T. Herve, S. Sanaur, R. M. Owens, G. G. Malliaras, Nat. Commun. 2013, 4:2133.

[45] P. Andersson, D. Nilsson, P. O. Svensson, M. Chen, A. Malmström, T. Remonen, T. Kugler, M. Berggren, Adv. Mater. 2002, 14, 1460.

[46] M. R. Abidian, D. H. Kim, D. C. Martin, Adv. Mater. 2006, 18, 405.

[47] E. W. Jager, O. Inganäs, I. Lundström, Science 2000, 288, 2335.

[48] X. Crispin, F. L. E. Jakobsson, A. Crispin, P. C. M. Grim, P. Andersson, A. Volodin, C. van Haesendonck, M. Van der Auweraer, W. R. Salaneck, M. Berggren, Chem. Mater. 2006, 18, 4354. 